\title{
Distribution géographique des populations de rôniers (Borassus aethiopum Mart, Arecaceae) et caractérisation phytoécologique de leurs habitats dans la zone soudano-guinéenne du Benin
}

\author{
GBESSO Florence ${ }^{1,2}$, YEDOMONHAN Hounnankpon ${ }^{2}$, TENTE Brice ${ }^{1}$, AKOEGNINOU Akpovi $^{2}$ \\ ${ }_{1}^{1}$ Laboratoire de Biogéographie et Expertise Environnementale (LABEE) ; Université d'Abomey-Calavi (UAC), BP 677 \\ Abomey-Calavi ; Bénin. Tel : 0022905347018/93937973, E-mail : viarrence1@yahoo.fr \\ 2 Laboratoire de Botanique et Écologie Végétale (LaBEV) ; Université d'Abomey-Calavi (UAC), \\ 01 BP 4521 Cotonou ; Tél. (229) 21360074 / Poste 127, E-mail : akoegnin@yahoo.fr / akoegnin@bj.refer.org
}

Original submitted in on 21st November 2013 Published online at www.m.elewa.org on 28 th February 2014. http://dx.doi.org/10.4314/jab.v74i1.14

\section{RESUME}

Objectif : L'étude a pour objectif d'étudier la distribution géographique et de réaliser la caractérisation phytoécologique des populations de rôniers (Borassus aethiopum) ainsi que leur caractérisation écologique dans la zone de transition.

Méthode et résultats : Le géo-référencement de toutes les localités abritant le rônier a été fait à l'aide des informations reçues dans les Centres Communaux de Production Agricoles (CECPA). Les inventaires floristiques et forestiers ont été réalisés à travers 70 placeaux rectangulaire de $50 \times 30 \mathrm{~m}$ dans trois communes des trois phytodistricts du secteur d'étude. B. aethiopum est plus distribué dans le district du Zou que dans les deux autres. Au total, 64 espèces ligneuses réparties en 25 familles sont inventoriées. Les groupes de relevé identifiés et caractérisés sont celui des champs et jachères ; celui des savanes boisées et celui des forêts galeries dégradées. Les paramètres floristiques et structuraux varient d'un groupement à l'autre. Ainsi, la richesse spécifique varie de 20 à 40 espèces, l'indice de diversité de Shannon de 1,89 à 5,49 bits, l'équitabilité de Pielou de 0,87 à 0,97 avec prédominance de la famille des Leguminosae. La densité des pieds adultes de B. aethiopum hectare oscille entre 77,78 et 132,89 tiges/ha et celle des pieds régénérés naturellement entre 1650 et 5236 tiges/ha. Quant à la surface terrière, elle est varié entre 26,47 à $29,55 \mathrm{~m}^{2} /$ ha et le diamètre moyen de l'arbre est compris entre 33,72 et $37,64 \mathrm{~cm}$ puis la hauteur entre 8 et $18 \mathrm{~m}$.

Conclusion et application: L'étude a permis de montrer que l'habitat naturel de Borassus aethiopum est la savane et la galerie forestière ainsi que la diversité des groupes de relevé abritant le rônier. La régénération naturelle de cette espèce aurait été une contribution à l'augmentation de la diversité biologique (vu son importance dans l'écosystème) mais elle est soumise à une forte pression anthropique. Ceci nécessite des actions urgentes de conservations. La plantation de l'espèce devient une nécessité pour sa domestication afin de permettre une exploitation durable vu qu'elle va utilisée à une grande échelle comme armature végétale 


\section{Gbesso et al. J. Appl. Biosci. 2014. Distribution géographique de Borassus aethiopum Mart et}

caractérisation phytoécologique de leurs habitats du Benin

dans les éléments en bétons et qu'elle représente une espèce à très lente croissance et à fort potentiel économique pour les populations de la zone soudano-guinéenne du Bénin.

Mots clés : Distribution spatiale, écologie, Borassus aethiopum, zone soudano-guinéenne du Bénin.

\section{ABSTRACT}

Objective The study aims to investigate geographical distribution of Palmyra (Borassus aethiopum) populations, to accomplish its phytoecological as well as the environmental characterization in the zone of transition Methods and Results: The geo-referencing of all localities with the Palmyra was done using information received at the Community Centers Agricultural Production (CECPA. Floristic and forest inventories were carried out through 70 rectangular plots of $50 \times 30 \mathrm{~m}$ in three municipalities of the three phytodistricts the study area. B. aethiopum was well distributed in the district of Zou than in the other two. A total of 64 woody species belonging to 25 families were inventoried. The identified and characterized groups of worth noting down were the fields and fallows; the wooded savannas and forests galleries. Floristic and structural parameters vary from one group to another. Thus, species richness ranges from 20 to 40 species, the Shannon diversity index of 1.89 to 5.49 bits, Pielou evenness of 0.87 to 0.97 with a predominance of the family Leguminosae. The density of adult feet $B$. aethiopum hectare ranges between 77.78 and 132.89 stems / ha and feet naturally regenerated between 1650 and 5236 stems / ha. As for the basal area is varied from 26.47 to $29.55 \mathrm{~m}^{2} /$ ha and the average diameter of the tree is between 33.72 and $37.64 \mathrm{~cm}$ and the height between 8 and $18 \mathrm{~m}$.

Conclusion and application: The study showed that the natural habitat of Borassus aethiopum is savanna and gallery forest and the diversity of groups identified housing the palmyra. Natural regeneration of this species has been contributing to the increase in biological diversity (given its importance in the ecosystem), but it is subject to high anthropogenic pressure. This requires urgent action conservations. Planting of species becomes a necessity for domestication to provide for sustainable exploitation because it will use on a large scale plant as reinforcement in concrete elements and it is a very slow growing species with high economic potential for the people of central Benin.

Keywords: Spatial distribution, ecology, African fan palm, ecological transition zone.

\section{INTRODUCTION}

Dans plusieurs pays en développement, les produits forestiers non ligneux (PFNL) ont été pendant longtemps sous-utilisés, mais ce n'est que depuis ces dernières années que le potentiel de les domestiquer pour accroître le bien-être des pauvres populations est devenu une préoccupation majeure (Leakey et al., 2005). Les informations disponibles sur les ressources forestières non ligneuses sont le plus souvent qualitatives et ne font pas ressortir les données quantitatives nécessaires pour démontrer les opportunités économiques ou pour le développement social et la gestion environnementale (Assogbadjo, 2003). Parmi ces espèces de la catégorie des PFNL, figure Borassus aethiopum (photos 1 et 2) qui est une espèce à usages multiples qui se rencontre dans les zones semi-arides et sub-humides d'Afrique tropicale, dans le Sud de l'Asie et dans les îles du pacifique et de
l'Océan Indien (Cabannes et al. , 1987). C'est une espèce des PFNL dont le principal produit très récolté est l'hypocotyle qui n'est rien d'autre que des fruits en début de germination, très appréciée des populations rurales et urbaines. La production d'hypocotyles se réalise en trois étapes essentielles : la collecte des fruits, qui commence en Janvier, le semis des fruits qui se fait à partir de Mars et la récolte des hypocotyles qui a lieu six à sept mois après le semis des fruits et rapporte un revenu consistant à ceux qui s'adonnent à l'activité ( Gbesso et al. , 2013). Beaucoup de travaux ont été menées pour contribuer à la mise en exergue de Borassus aethiopum dans la sous-région, principalement au Burkina, au Niger et en Côte d'lvoire. Les recherches entreprises sur l'espèce au Burkina ont permis de générer des informations sur la valeur alimentaire, économique et quelques modes de 


\section{Gbesso et al. J. Appl. Biosci. 2014. Distribution géographique de Borassus aethiopum Mart et}

caractérisation phytoécologique de leurs habitats du Benin

conservation de l'espèce (Yaméogo, 2007 ; Sogué, 2010 ; Kansolé, 2010). Les données disponibles sur l'écologie de $B$. aethiopum sont relatives à sa distribution et à sa biogéographie sur le continent africain (Cabannes et Chantry, 1987 ; Orwa et al., 2009). Au plan national, peu de travaux ont été exécutés pour la connaissance de l'espèce. Au nombre de ces auteurs, on peut citer ceux de Houankoun (2003), Wassi (2004), Kodjo (2005), Gibigaye et al. . (2008), Assogbadjo (2009), Hessou

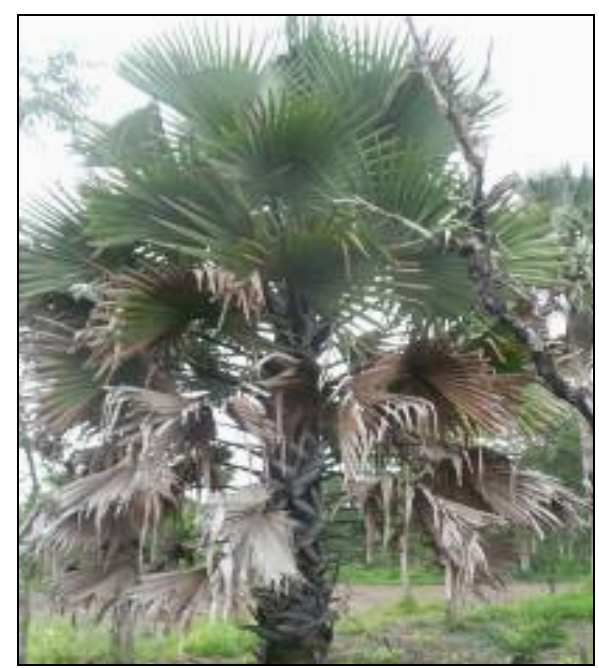

Photo 1: Vue d'un jeune rônier en croissance

\section{MATÉRIEL ET MÉTHODE}

Matériel : Le matériel utilisé dans le cadre de cette étude est constitué d'un ruban pour la délimitation des placeaux; de bande fluorescente pour matérialiser les limites des placeaux; de coupe-coupe pour l'ouverture des layons et la confection des piquets de coins; de sécateurs pour le prélèvement des échantillons; du ruban $\pi$ pour la mesure des diamètres des arbres à 1,30 $\mathrm{m}$ au-dessus du sol. Du clinomètre SUNNTO pour la mesure des hauteurs; des papiers journaux pour la confection des herbiers; du GPS (Global Positionning System) pour le géo-référencement des pieds du Borassus aethiopum et des sites échantillonnés ainsi que des fiches de relevé de végétation et une carte de situation géographique du secteur d'étude.

Milieu d'étude: Le milieu d'étude est la zone de transition soudano-guinéenne du Bénin selon le découpage de Adomou, 2005 comportant trois districts (Bassila, Borgou-Sud et Zou). Situé entre les parallèles $7^{\circ} 30^{\prime}$ et $9^{\circ} 45^{\prime}$ de latitude Nord et les méridiens $1^{\circ} 30$ et
(2011) et Ouinsavi al. (2011). Aucune de ces études n'a jusqu'à présent révélé la ni la distribution géographique de l'espèce ainsi que la caractérisation de son habitat. Notons aussi que seule la Flore Analytique du Bénin en a fait cas (Akoegninou et al., 2006). Cette étude permettra alors de disposer des données scientifiques pouvant permettre la gestion durable de l'espèce au Bénin et plus précisément dans la zone de transition soudano-guinéenne.

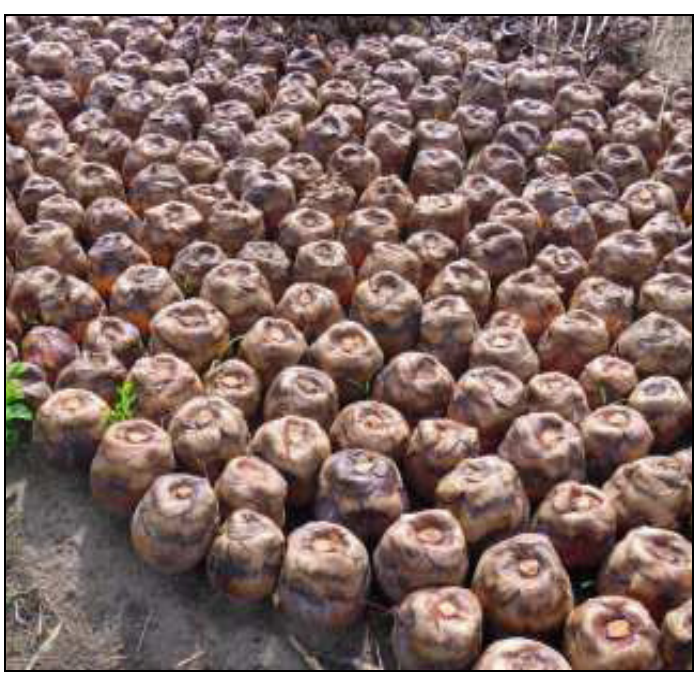

Photo 2 : Fruits et hypocotyles du rônier (Gbesso, Avril, 2012)

$2^{\circ} 40^{\prime}$ de longitude Est, il est limité au Nord par la zone soudanienne, au Sud par la zone guinéenne, à l'Ouest par la République du Togo et à l'Est par la République du Nigéria (Figure 1). Le secteur d'étude compte dix-huit (18) communes sur les 77 du pays. Le climat est de type tropical humide de transition avec u réseau hydrographique dendritique. On y rencontre des sols ferralitiques et des sols ferrugineux lessivés à concrétion sur roches cristallines. La végétation est constituée de forêt dense humide semi-décidue, de forêt dense sèche, de forêt claire et de savanes parsemés de forêts galerie (Akoegninou et al., 2006). La population est estimée à 2.244.873 habitants qui sont regroupés dans plusieurs groupes socioculturels dont les majoritaires sont les Tchabè, les Mahi, les Idaasha, les fon, les Bariba, les Dendi et les Cotocoly. L'agriculture est la principale activité qui occupe la population du secteur d'étude. (INSAE, 2002) 


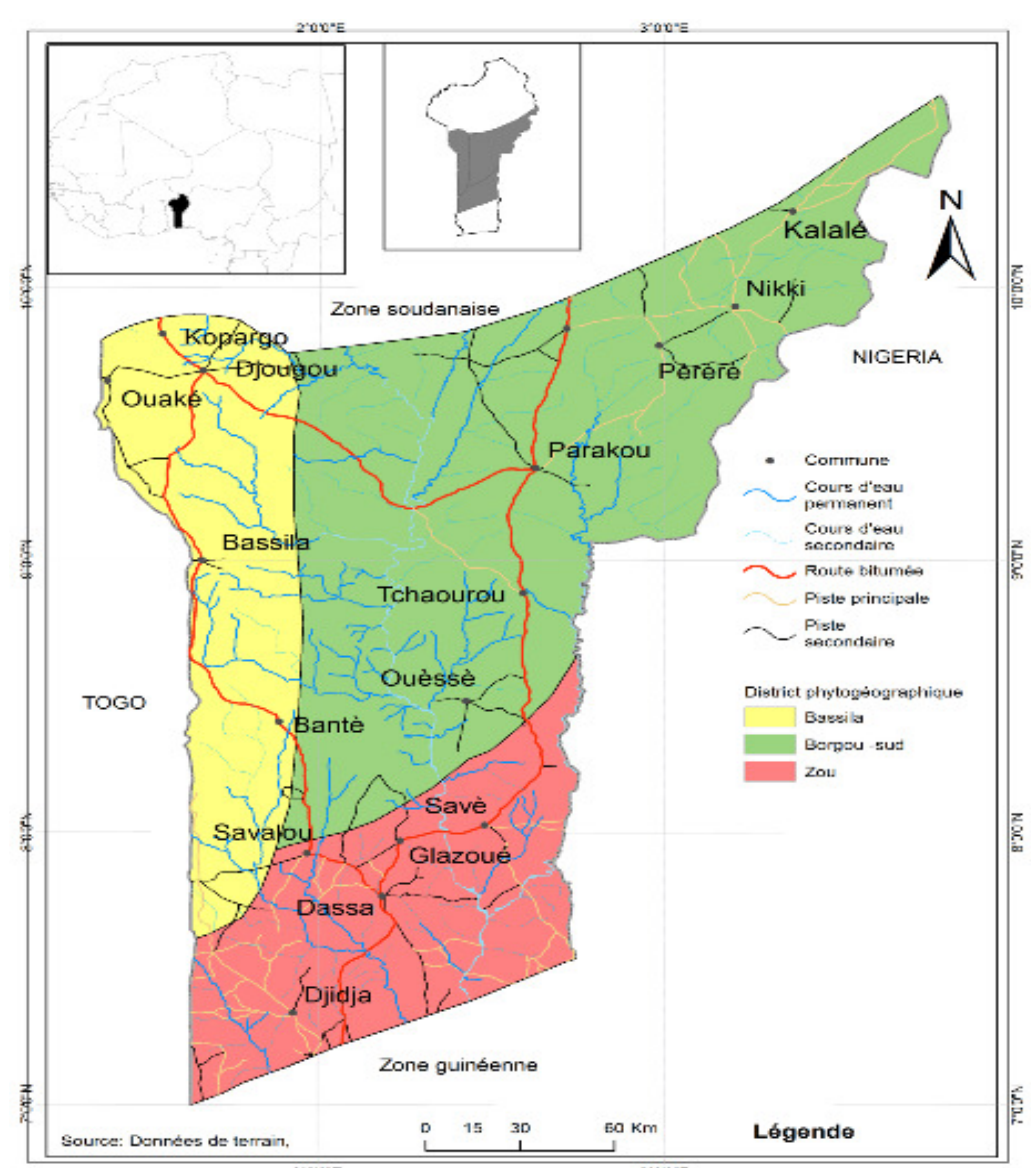

Figure 1: Situation du secteur d'étude. Source: adapté de Adomou, 2005

\section{Méthodes}

Collecte des données: Les données collectées ont porté sur la distribution spatiale et la densité d'une part et la caractérisation phytoécologique des communautés végétales abritant l'espèce d'autre part. S'agissant de l'étude de la distribution et de la densité du rônier, chacune des dix-huit communes du secteur d'étude a été explorée. Les coordonnées géographiques des localités de présence de l'espèce ont été systématiquement enregistrées à l'échelle communale. La caractérisation des habitats naturels de Borassus aethiopum a été réalisée dans trois communes, en raison d'une par district (Adomou, 2005) de la zone de transition du Bénin. Les choix de ces communes a été fait en fonction des informations reçues auprès des agents des CeCPA lors de l'exploration du secteur d'étude à des fins de distribution spatiale de $B$. aethiopum. Pour y parvenir, les relevés phytosociologiques ont été réalisés suivant la méthode sigmatiste de Braun-Blanquet (1932). Au niveau de chaque district, précisément dans les communes ciblées, l'inventaire des arbres et arbustes a été réalisé à l'intérieur des placeaux rectangulaires de $50 \mathrm{~m} \times 30 \mathrm{~m}$ $\left(1500 \mathrm{~m}^{2}\right)$ sur un transect de $8 \mathrm{~km}$ qui ont été disposés en tenant compte d'une distance de $2 \mathrm{~km}$ entre deux placeaux afin de rencontrer plusieurs types d'habitat de l'espèce. Les placeaux ont été installé à partir du centre du village en fonction des informations reçues auprès des populations, relatives à la présence de l'espèce. A chaque espèce est affecté le coefficient d'abondancedominance. L'échelle d'abondance dominance utilisée ici est celle de Braun-Blanquet (1932). Dans les placeaux, les données structurales et dendrométriques ont été relevées. Les espèces végétales ont été identifiées en partie sur le terrain à l'aide du document «Arbres, arbustes et lianes des zones sèches d'Afrique de l'Ouest» de Arbonnier (2002). Les espèces non identifiées sur le terrain ont été échantillonnées et déterminées par la suite à l'Herbier National du Bénin par comparaison aux spécimens de référence.

Traitement des données : Le tableau de contingence en présence-absence des espèces a été établi avec le tableur Excel à partir des données de relevés floristiques. 


\section{Gbesso et al. J. Appl. Biosci. 2014. Distribution géographique de Borassus aethiopum Mart et caractérisation phytoécologique de leurs habitats du Benin}

II a été soumis à la classification hiérarchique à l'aide du logiciel PC.ORD 5. 0 en utilisant la distance de Sørensen (Bray-Curtis) qui a permis la partition des relevés en groupes de relevés. L'« Indicator Species Analysis » a été utilisée pour calculer la valeur indicatrice de chaque espèce (Dufrêne et Legendre, 1997) et identifier numériquement les espèces caractéristiques de chaque groupe de relevé à partir du test de Monte Carlos ( $P$ value $<0,05)$. Ainsi les espèces indicatrices ont été utilisées pour nommer les groupes de relevé. La matrice relevés-espèces a ensuite servi à évaluer la diversité floristique par groupes de relevé identifiés et pour l'ensemble des trois communes du secteur d'étude. La diversité floristique des différents groupes de relevés a été évaluée à l'aide de la richesse spécifique, de la diversité en genres et de celle en familles (Daget, 1980). La nomenclature botanique utilisée est celle de la Flore Analytique du Bénin (Akoègninou et al., 2006). D'autres paramètres ont été également calculés pour caractériser les groupes de relevés obtenus. II s'agit de l'indice de diversité de Shannon-Wienner (H) (1949) et de l'équitabilité de Pielou (E) (Magurran, 2004). La structure du peuplement de $B$. aethiopum a été évaluée à l'aide de la surface terrière, la densité, le diamètre de l'arbre moyen et la hauteur du houppier.

- La densité du peuplement (N) correspond au nombre de tiges à l'hectare. Elle est obtenue par la formule :

$$
\mathbf{N}=\frac{\mathrm{n}}{\mathbf{3}}
$$

Où n est le nombre total d'individus d'arbres inventoriés dans le groupe de relevé et $S$ l'aire totale échantillonnée dans le groupe de relevé en hectare ;

- le diamètre de l'arbre moyen du groupement qui est déterminé par la formule :

$$
D g=\sqrt{\frac{\sum_{i}^{n} d i^{2}}{n}} \quad \text { exprimée en } \mathrm{m}^{2} / \mathrm{ha}
$$

avec $D=$ diamètre à hauteur de poitrine d'homme des arbres.

- la hauteur $\left(\mathrm{H}_{\mathrm{t}}\right)$ des individus de $B$. aethiopum est déterminée par la formule :

$$
\mathrm{H}_{\mathrm{t}}=\mathrm{Dv}(\tan V b+\tan V h) \text {; }
$$

Avec $\mathrm{Dv}$, la distance de visée correspondant à la distance de chute de l'arbre ;

$\mathrm{Vb}$, visée bas et $\mathrm{Vh}$, visée haut.
- La hauteur moyenne des individus du Vitellaria paradoxa par formation végétale est obtenue par moyenne arithmétique.

- L'indice de diversité de Shannon $(H)$ est donné par la formule :

\section{$\mathbf{H}=-\Sigma \mathbf{P i} * \log _{2} \mathbf{P i}$}

La diversité est faible lorsque $\mathrm{H}$ est inférieur à 3 bits, moyenne si $\mathrm{H}$ est compris entre 3 et 4 puis élevé quand $\mathrm{H}$ est supérieur ou égal à 4 bits (Legendre \& Legendre,1984; Frontier \& Piochod Viale, 1995).

- L'équitabilité de Pielou (E) traduit la manière dont les individus sont distribués à travers les espèces. Elle est maximale si les individus sont répartis de la même manière à travers les espèces. Elle varie de 0 (une espèce a une très forte abondance) à 1 (toutes les espèces ont la même importance). Elle se calcule par la formule suivante :

$$
E=H / \log 2(R s) \text {, }
$$

Où Rs désigne la richesse spécifique (Pielou, 1996). Les individus d'arbres dans chaque groupe de relevé ont été groupés en des classes de diamètre de $5 \mathrm{~cm}$ pour construire l'histogramme de la structure diamétrique de chaque groupe de relevé. La structure des groupe de relevés a été ajustée au modèle de Weibull à cause de sa grande flexibilité (Johnson \& Kotz, 1970 ; Bonou et al., 2009). Elle a été réalisée grâce au logiciel Minitab 14.0 et ajustée à la distribution de Weibull. La fonction de densité de probabilité de la distribution de Weibull est donnée par la formule :

$$
f(x)=\frac{c}{b}\left(\frac{x-a}{b}\right)^{c-1} \exp \left[-\left(\frac{x-a}{b}\right)^{c}\right]
$$

Où $x$ est le diamètre de l'arbre $i$, a est le paramètre de position (ici $a=10$ ) ; $b$ est le paramètre d'échelle ou de taille ; c'est le paramètre de forme lié à la structure observée. La caractérisation des peuplements est faite sur la base du coefficient de forme c (Bonou et al., 2009). Une analyse log-linéaire a été réalisée grâce au logiciel SAS (SAS Inc., 1999) pour tester l'adéquation du modèle de Weibull avec les structures diamétriques observées (Caswell, 2001).

Après avoir réparti les diamètres en différentes classes, un test de Kruskal-Wallis au seuil de $5 \%$ a été fait pour apprécier si la significativement des résultats d'un groupe de relevé à l'autre. 


\section{Gbesso et al. J. Appl. Biosci. 2014. Distribution géographique de Borassus aethiopum Mart et}

caractérisation phytoécologique de leurs habitats du Benin

\section{RÉSULTATS}

Distribution spatiale de Borassus aethiopum au centre du Bénin : Dans le secteur d'étude, les localités de Savè et Glazoué sont des zones à forte prédominance de rônier (Tableau 1). De l'analyse du tableau 1, il ressort

que de façon générale $B$. aethiopum est plus abondante dans le phytodistrict de Zou que dans les deux autres de la zone de transition soudano-guinéenne

Tableau 1 : Fréquence relative de B. aethiopum par District phytogéographique

\begin{tabular}{|l|l|l|l|}
\hline District phytogéographique & Bassila & Borgou-Sud & Zou \\
\hline Densité & $54-126$ & $55-152$ & $63-266$ \\
\hline
\end{tabular}

Source : Résultat d'enquête, avril 2012.

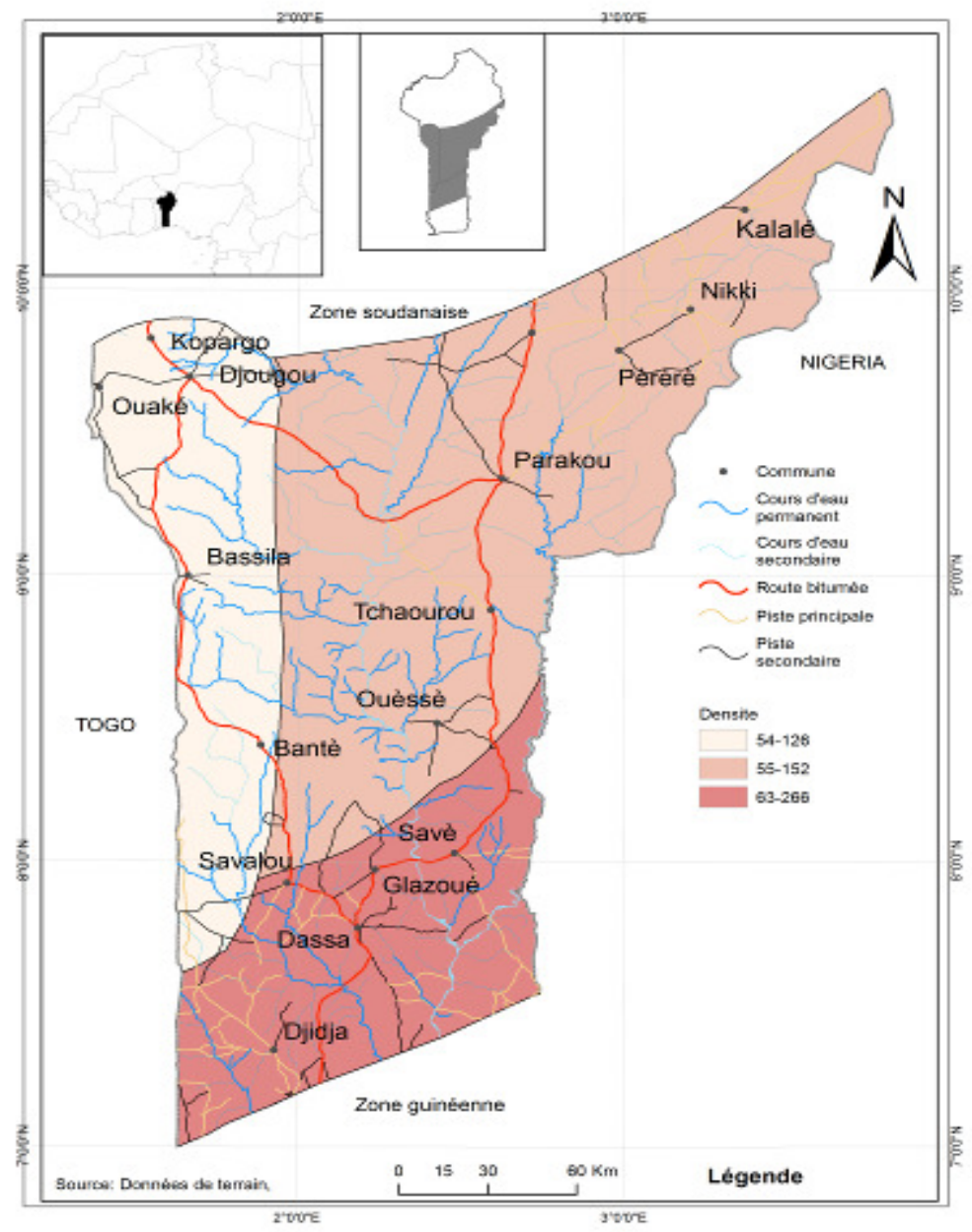

Figure 2: Distribution spatiale de $B$. aethiopum au centre Bénin

Caractérisation des groupes de relevé sous le Borassus aethiopum

Partition des relevés au sein des formations végétales : La matrice brute constituée de 70 relevés et de 64 espèces est soumise à une analyse multivariée par le biais de la DCA (Detrended Correspondence Analysis). Les axes factoriels de la DCA expliquent 2,69\% de I'inertie totale. La figure 3 présente l'ordination des relevés sur le plan des axes 1 et 2 de la DCA. 


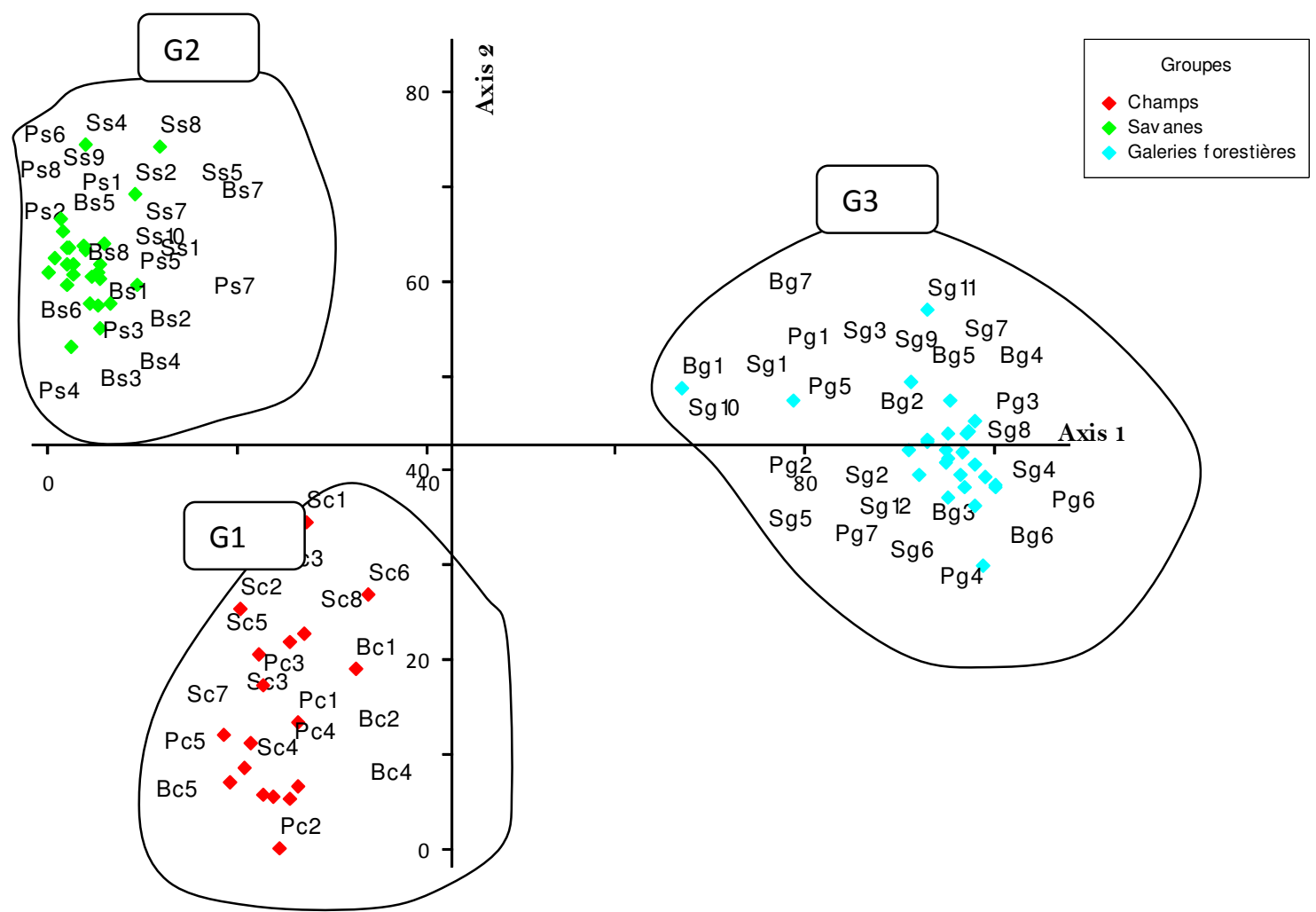

Figure 3 : Répartition des relevés dans les plans factoriels des axes 1 et 2 de la DCA

Trois groupes de relevés sont distingués. II s'agit de : G1: constitué des relevés effectués dans les champs et jachères ; $G 2$ : réunissant les relevés effectués dans les savanes et G3 : formé des relevés effectués dans les galeries forestières. L'axe 1 discrimine à son origine les relevés de savanes et à son extrémité droite les relevés de forêts galerie. II traduit alors un gradient topographique. L'axe 2 oppose les relevés des champs et jachères (à son origine) aux relevés de savanes (à son extrémité droite). II indique un gradient d'anthropisation de la végétation. Le dendrogramme des relevés (figure 4) confirme la partition des relevés en 3 groupes à $51 \%$ de dissemblance. 


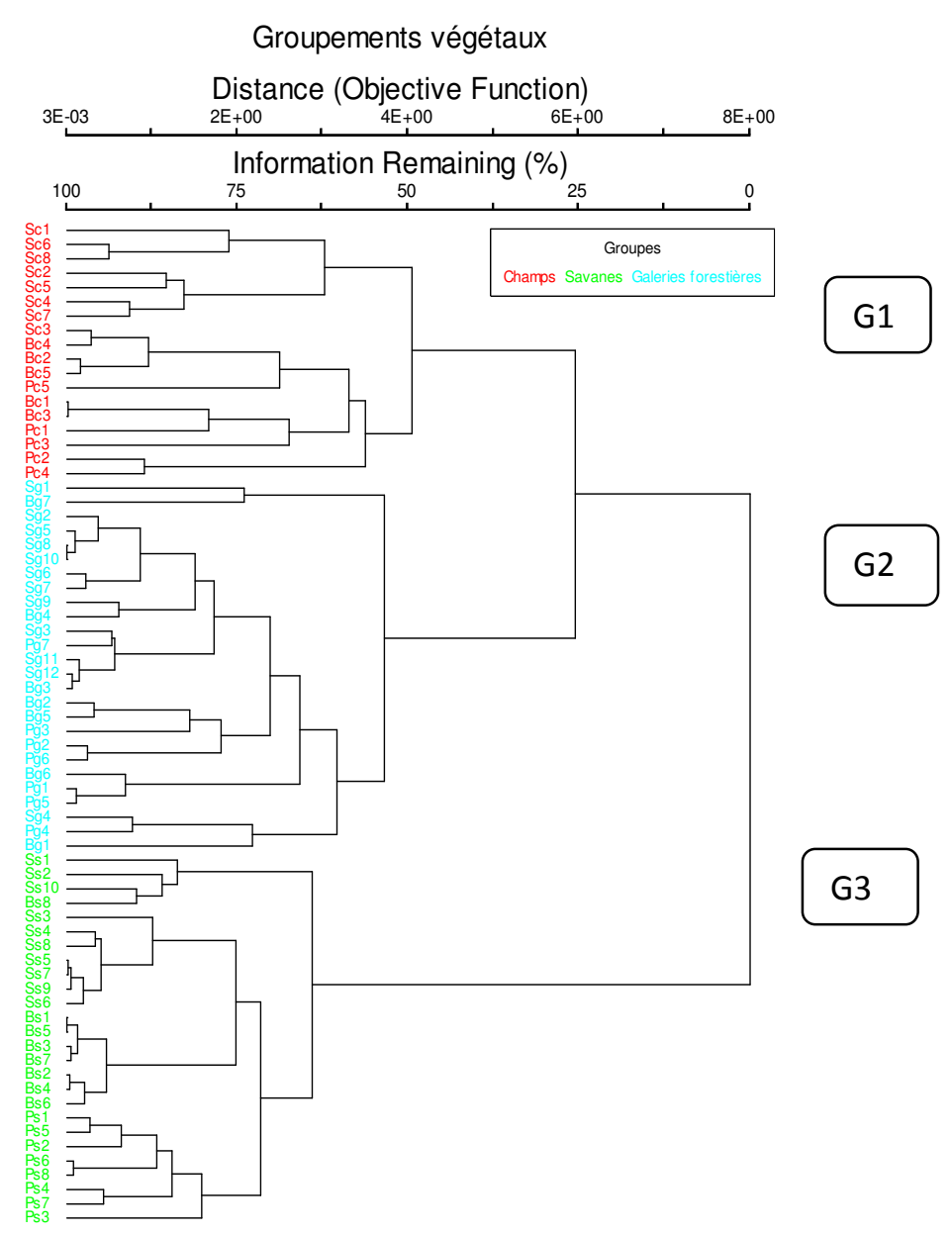

Figure 4 : Dendrogramme de dissimilarité des trois groupes de 70 relevés

Composition floristique et diversité spécifique des groupes de relevé : Au total 62 espèces ont été recensées. Elles sont réparties en 25 familles dont les plus importantes sont les Leguminosae ( 15 espèces), les Combretaceae (7 espèces), les Rubiaceae ( 6 espèces) et les Meliaceae (4 espèces). Le tableau 2 résume les informations sur la composition floristique et la diversité spécifique des groupes de relevé enregistrés dans le milieu d'étude. II ressort de l'analyse du tableau 2 que la richesse spécifique des groupes de relevé varie de 20 à 40 espèces végétales. Du point de vue de la diversité spécifique la communauté végétale $\mathrm{G} 2$ présente l'indice de diversité de Shannon le plus élevé ; soit 5,49 bits et une équitabilité de 0,97 . Cette valeur d'équitabilité indique une bonne répartition des espèces et une bonne exploitation des ressources du milieu par les espèces.
L'indice de diversité de Shannon des deux autres groupes de relevé est également supérieur à 3,5 bits $(\mathrm{G} 1=3,89$ et $\mathrm{G} 3=4,21)$. Quant à l'équitabilité de Pielou, elle varie entre 0,84 et 0,90 pour le groupement ; on peut alors conclure que les espèces du groupement $\mathrm{G} 1$ sont moins bien réparties que celles des groupements $\mathrm{G} 2$ et G3. De cette analyse, on peut donc déduire que les habitats préférés de $B$. aethiopum sont les savanes, précisément les savanes humides et les galeries forestières.

Caractéristiques structurales et dendrométriques de Borassus aethiopum : Les caractéristiques structurales et dendrométriques de Borassus aethiopum varient significativement d'un groupe de relevé à un autre (Tableau 3). 


\section{Gbesso et al. J. Appl. Biosci. 2014. Distribution géographique de Borassus aethiopum Mart et}

caractérisation phytoécologique de leurs habitats du Benin

Tableau 2 : Caractéristiques et diversité de communautés identifiées

\begin{tabular}{|c|c|c|c|c|c|c|c|c|}
\hline Code & $\begin{array}{ll}\text { Nom } & \text { du } \\
\text { groupe } & \text { de } \\
\text { relevé } & \end{array}$ & $\begin{array}{l}\text { Espèces } \\
\text { indicatrices }\end{array}$ & $\begin{array}{l}\text { Valeur } \\
\text { indicatrice }\end{array}$ & $\begin{array}{l}\text { P Value } \\
\text { (Monte } \\
\text { Carlos } \\
\text { test) }\end{array}$ & $\begin{array}{l}\text { Richesse } \\
\text { spécifique }\end{array}$ & $\begin{array}{l}\text { Familles } \\
\text { dominantes }\end{array}$ & $\begin{array}{l}\text { Indice de } \\
\text { Shannon }\end{array}$ & $\begin{array}{l}\text { Equitabilité } \\
\text { de Piélou }\end{array}$ \\
\hline $\mathrm{G} 1$ & $\begin{array}{l}\text { Champs et } \\
\text { jachères }\end{array}$ & $\begin{array}{l}\text { Adansonia } \\
\text { digitata } \\
\text { Mangifera } \\
\text { indica } \\
\text { Anacardium } \\
\text { Occidentale }\end{array}$ & $\begin{array}{l}94,4 \\
88,9\end{array}$ & $\begin{array}{l}0,0002 \\
0,0002\end{array}$ & 20 & $\begin{array}{l}\text { Leguminosae } \\
\text { et } \\
\text { Rubiaceae }\end{array}$ & 3,89 & 0,90 \\
\hline G2 & $\begin{array}{l}\text { Savanes } \\
\text { boisées }\end{array}$ & $\begin{array}{l}\text { Combretum } \\
\text { molle } \\
\text { Burkea } \\
\text { africana }\end{array}$ & $\begin{array}{l}100,0 \\
100,0\end{array}$ & 0,0002 & 40 & $\begin{array}{l}\text { Combretaceae } \\
\text { et } \\
\text { Leguminosae }\end{array}$ & 5,49 & 0,97 \\
\hline G3 & $\begin{array}{l}\text { Galeries } \\
\text { forestières } \\
\text { dégradée }\end{array}$ & $\begin{array}{l}\text { Bambusa } \\
\text { vulgaris } \\
\text { Elaeis } \\
\text { guineensis } \\
\text { Raphia } \\
\text { hookeri }\end{array}$ & 92,3 & 0,0002 & 31 & $\begin{array}{l}\text { Leguminosae } \\
\text { et } \\
\text { Rubiaceae }\end{array}$ & 4,21 & 0,84 \\
\hline
\end{tabular}

Tableau 3 : Paramètres structurales et dendrométriques de $B$ aethiopum dans les différents groupes de relevé d'étude

\begin{tabular}{|c|c|c|c|}
\hline \multirow[t]{2}{*}{ P Paramètres } & \multicolumn{3}{|c|}{ Groupes de relevés } \\
\hline & G1 & G2 & G3 \\
\hline $\mathrm{Dg}(\mathrm{cm})$ & 36,83 & 37,64 & 33,72 \\
\hline G (m²/ha) & 28,93 & 29,55 & 26,47 \\
\hline N (tiges/ha) & 77,24 & 132,89 & 110,78 \\
\hline Hauteur (m) & 10 & 14 & 12 \\
\hline
\end{tabular}

Soit $D g=$ diamètre de l'arbre moyen, $\mathrm{G}\left(\mathrm{m}^{2} / \mathrm{ha}\right)=$ la surface terrière, $N=$ densité et $\mathrm{G}=$ Groupe de relevé

L'analyse des paramètres dendrométriques de $B$. aethiopum dans la zone de transition soudano-guinéenne (champs/jachères; savanes et galeries forestières) indique une différence significative pour les diamètres, les hauteurs et les densités des arbres entre les trois groupes $(p<0,05)$. La structuration des moyennes indique que le diamètre moyen de l'arbre dans les champs est différent de celui des savanes et des galeries forestières. Il est plus élevé dans les savanes $(37,64 \mathrm{~cm})$ suivi des champs/jachères $(36,83 \mathrm{~cm})$ et des galeries forestières $(33,72 \mathrm{~cm})$. De même, c'est dans les savanes qu'on retrouve les individus les plus grands (hauteur 


\section{Gbesso et al. J. Appl. Biosci. 2014. Distribution géographique de Borassus aethiopum Mart et caractérisation phytoécologique de leurs habitats du Benin}

moyenne $=14 \mathrm{~m}$ ). Quant à la densité du peuplement, les plus élevées sont ainsi observées dans les savanes (132,89 arbres/ha) tandis que les plus faibles valeurs de ces densités ont été observées dans les champs/jachères ( 77,24 tiges / ha).

Structure diamétrique du Borassus aethiopum au sein des différentes communautés végétales: La répartition du Borassus aethiopum par classe de diamètre au sein des différents groupes de relevé est présentée par la figure 5 . II est à constater que quel que soit le groupe considéré, les effectifs du Borassus aethiopum sont plus élevés dans les classes de diamètre de] $30 ; 40$ $\mathrm{cm}]$. Les effectifs les plus faibles sont constatés dans les classes de] $20 ; 30 \mathrm{~cm}]$ et] $45 ; 50 \mathrm{~cm}]$.
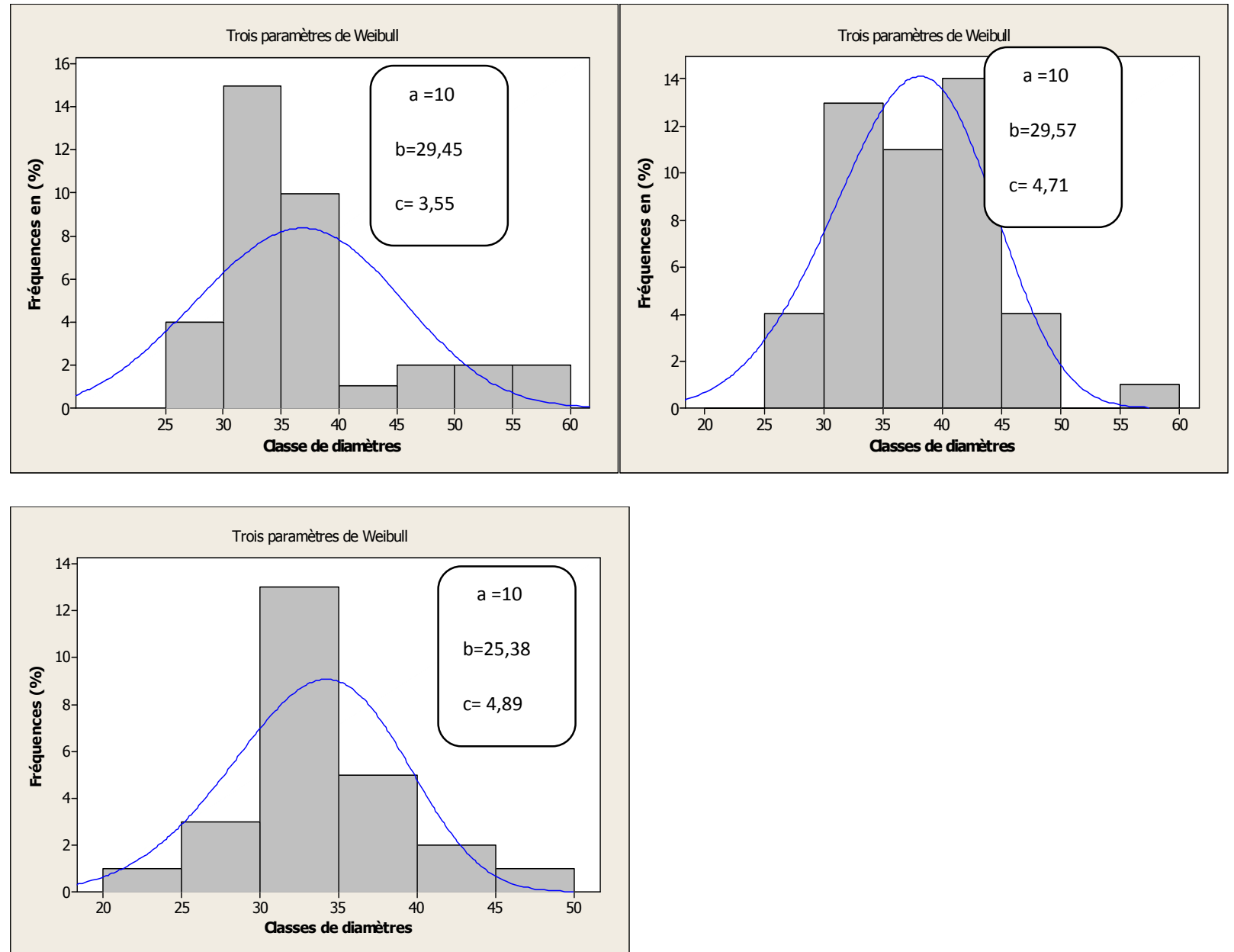

Figure Error! No text of specified style in document.: Structure diamétrique de $B$. aethiopum dans les communautés végétales $a$ : champs et jachères, $b$ : savanes, $c$ : forêt galerie

II ressort également de l'analyse de la figure 5 que les individus de tous les groupes de relevés se retrouvent dans la classe de diamètre située entre] $30 ; 40 \mathrm{~cm}$ ], ce qui confèreà ces groupes une structure asymétrique centrée. L'ajustement de la structure à la fonction mathématique de Weibull donne un paramètre de forme $c=1,48$ caractéristique des peuplements avec prédominance d'individus jeunes ou de faible diamètre. Les individus de très gros diamètre sont quasi inexistants au niveau des trois (03) groupements. Le test de Kruskal-Wallis révèle qu'il existe une différence significative entre les diamètres de Borassus aethiopum des différents groupes de relevé $(H=8,45 ; D F=2 ; P=0,015)$ 


\section{Gbesso et al. J. Appl. Biosci. 2014. Distribution géographique de Borassus aethiopum Mart et}

caractérisation phytoécologique de leurs habitats du Benin

\section{DISCUSSION}

La famille des Arecaceae est apparue au Crétacé et prit rapidement une grande extension, comme le montrent les abondants restes fossiles du Tertiaire (Cabannes et Chantry, 1987). Selon les mêmes auteurs, la répartition géographique des espèces et genres de la famille des Arecaceae est le résultat des fluctuations paléogéographiques qui ont réduit considérablement l'aire de ces dernières. Ainsi, on rencontre le $B$. aethiopum dans les zones soudaniennes et soudanosahéliennes. II a été introduit par les hommes dans certaines savanes pyrophiles de l'intérieur et dans les savanes du littoral atlantique, au Ghana, Côte d'Ivoire, Bénin, Gabon, Congo. C'est surement ce qui justifie la présence de l'espèce au Bénin et surtout dans le centre qui regorge de vastes étendues de savanes bien drainés. Les résultats de la présente recherche montrent que le $B$. aethiopum se rencontrent dans les savanes et les galeries forestières. Ces résultats confirment en partie ceux de Souza (1980) qui stipulent que l'espèce est surtout rencontrée dans les savanes. L'étude menée sur la caractérisation structurale de $B$. aethiopum indique quatre grands faciès de groupes végétaux autour desquels se fixe Borassus aethiopum. Au nombre de ces groupes, seuls ceux des formations végétales des savanes et des parties situées le long des cours d'eau ou parties périodiquement inondées permettent un bon développement de l'espèce. Ces aspects phytosociologiques peuvent être justifiés par le fait que Borassus aethiopum n'est pas domestiqué et est aussi exigent en fonction des conditions climatiques. Ces résultats confirment ceux de Mathias (2004) qui a su montré les conditions de développement phytosocilogique de Borassus aethiopum. Les familles botaniques les plus riches en espèces sont les Leguminosae et les Combretaceae. La prédominance des Leguminosae n'est une caractéristique de la zone soudano-guinéenne mais une constante de la flore du Bénin (Akoègninou, 2006). S'agissant des Combretaceae, la majorité des taxons appartenant à cette famille sont des savanes. En ce qui concerne la surface terrière des groupes végétaux, elle varie entre 0.08 et $0,12 \mathrm{~m} 2 / \mathrm{ha}$. Ceci laisse voir l'importance dans l'exploitation de Borassus aethiopum en arboriculture. Le nombre de pieds par hectare oscille entre 78 et 133 pour les pieds adultes et entre 5236 et 1650 pour les pieds régénérés naturellement. $A$ première vue, les valeurs des densités des pieds régénérés semblent satisfaisantes, mais il est indispensable de noter que ces pieds n'ont pas encore de tronc et que suivant les étapes de croissance de l'espèce, les tiges n'apparaissent qu'après 6 à 8 ans au moins. Ainsi, plus des $3 / 4$ de ces pieds régénérés naturellement disparaissent à cause, non seulement des perturbations naturelles (concurrence entre les pieds adultes et les pieds jeunes...) mais aussi des effets anthropiques (feux de végétation, agriculture, exploitation forestière...). Ces résultats indiquent que le peuplement est très dense dans les savanes humides que dans les formations anthropisées; ce qui peut faire dire que l'espèce est menacée de disparition. De même, c'est dans les savanes qu'on retrouve les individus les plus grands (hauteur moyenne $=14 \mathrm{~m}$ ). Ces résultats sont un peu différents de ceux de Hessou (2011) et de Ouinsavi (2011) qui ont obtenu respectivement plus de $15 \mathrm{~m}$ de hauteur dans la zone soudanienne du pays. Cela pourrait s'expliquer par la présence élevée de compétition entre les espèces pour la recherche de l'énergie solaire; ce qui pourrait inhiber la croissance en hauteur de ces individus observés dans la zone soudano-guinéenne. En milieux anthropisés (champs) par contre, la rôneraie est clairsemée et éparse par endroits donc bien ensoleillée. Les faibles valeurs observées pour la hauteur et le diamètre en agrosystème s'expliquent par le fait que les formations naturelles ont été progressivement transformées en champ de cultures et par conséquent fortement anthropisées. Aussi, dans les écosystèmes fortement colonisés, l'arbre est régulièrement élagué et ses organes prélevés à diverses fins. Ce qui peut induire un impact négatif sur ses caractéristiques dendrométriques. Bayer \& Water-Bayer (1999) ont noté que l'élagage a un impact sur la croissance des arbres. Selon ces auteurs, l'élagage réduit la quantité de feuilles, inhibant ainsi la photosynthèse qui agit sur la croissance des arbres. De plus, Sinsin et al. (2004) ont montré que dans les différentes zones climatiques du Bénin, plus fortes sont les pressions exercées sur les individus d'Afzelia africana, plus faibles sont les hauteurs des arbres. De même, une étude menée par Assogbadjo et al. (2010) dans la forêt classée de Wari-Maro a montré que les caractéristiques dendrométriques ont les plus grandes valeurs pour Anogeissus leiocarpa dans les peuplements soumis à une faible pression. De tels résultats ont été obtenus par Kiki (2008) sur Vitex doniana, Fandohan et al. (2010) et Nyadoi (2005) sur le tamarinier qui ont montré que les pressions anthropiques ont un effet négatif sur les paramètres dendrométriques tels que la densité de régénération et la densité des adultes mais un effet positif sur le diamètre moyen. $\mathrm{Ce}$ qui confirme aussi les densités relativement plus faibles 
observées dans les écosystèmes perturbés étudiés et le diamètre moyen élevé en terroir riverain. De l'avis des populations, lors des défriches, beaucoup de plantules sont déracinées au profit des cultures ; ce qui contribuerait aussi à réduire considérablement la densité de régénération dans ces écosystèmes. Cet avis est bien partagé par Abotchi (2002), Dan et al. (2010) qui ont montré que dans certaines localités, le faible nombre des plantules peut résulter des défrichements agricoles intensifs, l'exploitation pour le fourrage, l'exploitation du bois d'œuvre et bois-énergie et la fabrication du charbon de bois. Pour le diamètre moyen, on constate qu'il n'y a pas une nette démarcation entre les groupes végétaux et varie de 32,51 à $38,41 \mathrm{~cm}$. L'ajustement de la structure à la fonction mathématique de Weibull donne un paramètre de forme $c=1,48$ caractéristique des peuplements avec prédominance d'individus jeunes ou de faible diamètre. II est indispensable de noter que sur Borassus aethiopum, les individus ayant un gros diamètre sont les jeunes pieds alors que les individus de faible diamètre représentent les vieux pieds. II ressort de ce constat que les populations de Borassus aethiopum enregistrées dans le secteur d'étude sont de vieux pieds. On peut donc déjà conclure que la zone de transition soudano guinéenne regorge plus de vieux pieds de Borassus aethiopum que de jeunes ; il se pose donc le problème de renouvellement

\section{CONCLUSION}

Cette étude sur la distribution spatiale et la caractérisation écologique des peuplements du Borassus aethiopum dans la zone de transition, constitue une contribution à la connaissance de l'espèce et aux paramètres écologiques indispensables à sa conservation dans cette partie du Bénin. La caractérisation phytosociologique a permis d'identifier trois (03) grands groupes de peuplements distincts par leurs traits spécifiques induits par la topographie et les des populations de rônier afin de permettre sa conservation dans les formations végétales dans le secteur d'étude en particulier et au Bénin en général. En effet, les plus gros effectifs ont été rencontrés en galeries forestières et entre les classes de diamètre de] $30 ; 40$ $\mathrm{cm}]$ alors que les plus faibles ont été retrouvés dans les champs et entre les classes de diamètre de]20;30 cm] et] $40 ; 50 \mathrm{~cm}$ ]. A ceci, doit être ajouté le fait que, plus le rônier prend de l'âge, plus son tronc se rétrécie. Ces résultats indiquent que le peuplement est très dense selon que l'activité anthropique est moins importante ainsi que la compétition entre les végétaux et plus précisément entre les pieds adultes et les régénérés; ce qui pourrait réduire l'importance des individus observés dans les formations de champs où l'action anthropique est permanente. En milieu de galeries forestières par contre, les conditions naturelles favorisent la régénération de l'espèce. Les résultats de la présente étude confirment en partie ceux de Ouinsavi et al. , (2011) qui pensent que selon Giffard, (1967), cet état de choses est dû à l'influence du climat sur les individus de l'espèce et aussi à la compétition existante aux pieds des rôniers adultes qui empêche le développement des jeunes semis (UNSO, 1993). Selon le même auteur, cela s'explique par la concurrence des racines vis-à-vis de l'eau en saison sèche.

strates végétatives que sont les champs, les savanes et des forêt galeries. On peut retenir que deux types d'habitat sont favorables au développement de l'espèce. II s'agit des zones de savanes et des galeries forestières. En outre, on peut également retenir que l'espèce est pratiquement présente dans toutes les communes de la zone de transition mais en densité très variée; et que c'est à Savè et à Glazoué qu'elle est assez abondante et fait plus objet de commercialisation.

\section{REMERCIEMENTS}

Cette étude a bénéficié d'un appui financier du Ministère de l'Enseignement Supérieur et de la Recherche du Bénin à qui les auteurs expriment leur profonde gratitude.

\section{RÉFÉRENCES BIBLIOGRAPHIQUES}

Abotchi T. (2002) : Colonisation agricole et dynamique de l'espace rural au Togo: cas de la plaine septentrionale du Mono. Revue du C.A.M.E.S, Sciences Sociales et Humaines, 4(1), pp97-108.

Adomou C. A. (2005): Vegetation Patterns and Environmental gradients in Benin. Implications for biogeography and conservation. PhD Thesis; Wageningen University, Wageningen: 133p

Akoegninou A., Adjakidje V., Essou J-P., Sinsin B., Yedomonhan H., W. J. Van Der Brug, L. J. G. Van Der Maesen, (2006): Flore analytique du Bénin. Université d'Abomey-Calavi, Cotonou, Bénin, $1034 \mathrm{p}$. 


\section{Gbesso et al. J. Appl. Biosci. 2014. Distribution géographique de Borassus aethiopum Mart et}

caractérisation phytoécologique de leurs habitats du Benin

Arbonnier M., (2002) : Arbres, arbustes et Lianes des zones sèches d'Afrique de l'Ouest. CIRAD; $\mathrm{MNHN}$; UICN ; 2ème édition, Artecom (89, Pont-sur-moyenne), France 573p.

Assogbadjo, A. E. (2006). Importance socio-économique et étude de la variabilité écologique, morphologique, génétique et biochimique du baobab (Adansonia digitata L.) au Bénin. Thèse de doctorat. Faculty of Bioscience Engineering, Ghent University, Belgium. 213 p.

Bayer W. \& Waters-Bayer A. (1999) : La gestion des fourrages. CTA Wageningen. The Netherlands: $246 p$.

Braun-Blanquet J., (1932) : Plant sociology. The study of plant communities. Ed. McGray Hill, New-York, London. $439 \mathrm{p}$.

Cabannes Y. et Chantry G., (1987): Le rônier et le palmier à sucre dans l'habitat. Edition GRET (France) $90 \mathrm{p}$.

Dan G., Ali Mahamane et Karimou Jean Marie Ambouta (2010) : Peuplement des parcs à Neocarya macrophylla (Sabine) Prance et à Vitellaria paradoxa (Gaertn. C.F.) dans le sud-ouest nigérien : diversité, structure et régénération ; Int. J. Biol. Chem. Sci. 4(5), pp1706-1720.

Fandohan A. B., Assogbadjo A. E., Glèlè kakaï R. L., Sinsin B. \& Van Damme P. (2010): Impact of habitat type on the conservation status of tamarind (Tamarindus indica L.) populations in the W National Park of Benin. Fruits, 65, pp1119.

Gbaguidi S. V., Gbaguidi Aisse G., Gibigaye M., Adjovi E. C., Amadji A., Sinsin B. (2010) : Association béton-bois de Borassus aethiopum pour la réalisation des éléments fléchis faiblement chargés et des raidisseurs des murs porteurs : caractéristiques physico-mécaniques du bois de rônier, Actes de colloque scientifique de Ouagadougou, 10p.

Kiki M. (2008) : Structure et régénération naturelle des populations de Tamarindus indica L. et de Vitex doniana Sw. dans la Réserve de Biosphère Transfrontalière du W/Bénin : Cas de la Commune de Banikoara. Mémoire d'Ingénieur des travaux. EPAC/UAC : 89p.

Hoyt, E. (1992) : La conservation des plantes sauvages apparentées aux plantes cultivées. IBPGR. UICN. WWf. BRG. 49p.

MATHIAS M., (2004) : L'utilisation durable des palmiers Borassus aethiopum, Elaeis guineensis et
Raphia hookerie

http/l:www.google.fr/searchq=cache:bNIXRX1jF FJ:http://www.gtz/:de/TOEB L\%

Ouinsavi C., Gbemavo C., Sokpon N. (2011): Ecological structure and fruit production of African fan palm (Borassus aethiopum) populations American journal of plant sciences, 2, pp733-743. doi:10.1016/i.foreco.2004.10.069

Shannon C. E. (1948): A mathematical theory of communications. Bell Syst. Techn. J., 27: pp623-656.

Sinsin B., Eyog Matig O., Assogbadjo A. E., Gaoué O. G. \& Sinadouwirou T. (2004): Dendrometric characteristics as indicators of pressure of Afzelia africana Sm. Dynamic changes in trees found in different climatic zones of Benin. Biodiversity and conservation, 13, pp1555-1570. 